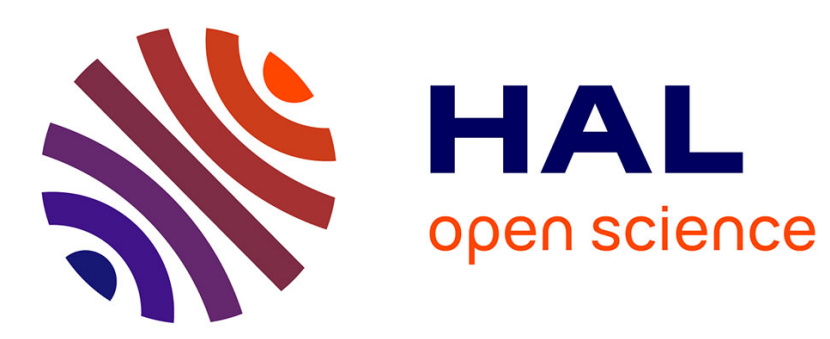

\title{
Uncertainty Quantification With Ensemble-Based FWI - Application to 2D OBC Data from the Valhall Field
}

Julien Thurin, Romain Brossier, Ludovic Métivier

\section{To cite this version:}

Julien Thurin, Romain Brossier, Ludovic Métivier. Uncertainty Quantification With Ensemble-Based FWI - Application to 2D OBC Data from the Valhall Field. 81st EAGE Conference and Exhibition 2019, Jun 2019, London, United Kingdom. 10.3997/2214-4609.201900881 . hal-02325630

\section{HAL Id: hal-02325630 \\ https://hal.science/hal-02325630}

Submitted on 24 Nov 2020

HAL is a multi-disciplinary open access archive for the deposit and dissemination of scientific research documents, whether they are published or not. The documents may come from teaching and research institutions in France or abroad, or from public or private research centers.
L'archive ouverte pluridisciplinaire HAL, est destinée au dépôt et à la diffusion de documents scientifiques de niveau recherche, publiés ou non, émanant des établissements d'enseignement et de recherche français ou étrangers, des laboratoires publics ou privés. 


\title{
Uncertainty quantification with ensemble-based FWI - application to 2D OBC data from the Valhall field
}

\author{
J.Thurin ${ }^{1}$, R. Brossier ${ }^{1}$, L. Métivier ${ }^{2,1}$ \\ ${ }^{1}$ Univ. Grenoble Alpes, ISTerre, F-38000 Grenoble, France \\ ${ }^{2}$ Univ. Grenoble Alpes, CNRS, LJK, F-38000 Grenoble, France
}

January 14, 2019

\begin{abstract}
Full Waveform Inversion (FWI) is an ill-posed nonlinear inverse problem, which aims at recovering high-resolution geophysical subsurface models. This work focuses on the joint application of the Ensemble Transform Kalman Filter and Quasi-Newton Full Waveform Inversion (ETKF-FWI) on seismic-exploration field-data to estimate uncertainty. We reintroduce the ETKF-FWI formalism under a compact form and apply our scheme on a 2D line from the 3D OBC data from the North Sea Valhall field. This scheme allows us to express our solution as an ensemble of subsurface parameter models in a Bayesian formalism. The mean of the output ensemble yields a parameter estimation similar in quality to that of standard adjoint-based FWI, and the posterior covariance of the ensemble holds the uncertainty of the parameter estimation as well as resolution information. We show that both the uncertainty estimation and the resolution information are consistent with our understanding of the FWI problem. This application demonstrates the suitability of ETKF-FWI for uncertainty estimation in full waveform tomography beyond synthetic benchmarks.
\end{abstract}




\section{Introduction}

Full Waveform Inversion (FWI) is an ill-posed nonlinear inverse problem, which aims at recovering highresolution geophysical subsurface models (Virieux and Operto, 2009; Virieux et al., 2017). FWI is solved by iteratively improving the data-fit between synthetic and observed waveform data, generally recorded at the surface. The approach is now widely used, both in academia and industry, and the output models might be used for direct interpretation or further processing (migration). Confidence in the solution of the FWI problem is of crucial importance for better geodynamic/tectonic understanding as well as industrial decision-making. Up to recent years, however, the FWI problem has been mainly solved under a deterministic local optimization framework: its solution is highly dependent on settings such as initial model quality, data pre-processing, and hyper-parameters associated with regularization and preconditioning for instance. Within this computationally expensive large-scale optimization framework, uncertainty estimation is generally omitted or limited to quality control (data fit, wavelet consistency), comparison and/or cross-validation with other geophysical methods or observables (well-log, CIG flatness).

However, recent methodological propositions, often based on the Bayesian inference framework (Tarantola, 2005), have demonstrated the interest of uncertainty estimation for the FWI community (Fichtner and van Leeuwen, 2015; Fang et al., 2018). Despite not being broadly adopted, uncertainty estimation methodologies are a step in the right direction for a better general solution of the FWI problem.

Inspired by the Data Assimilation (DA) tools, which have been designed to tackle large-scale, non-linear system state estimation problems, we have proposed an alternative method for uncertainty quantification (Thurin et al., 2017), based on mixed Ensemble Transform Kalman Filter (ETKF, Bishop et al., 2001) and Quasi-Newton FWI scheme. ETKF has been selected, as it has proven to be efficient at operational scale for application in several areas of research as well as embedding uncertainty estimation directly in its solutions. Our resulting ETKF-FWI scheme allows us to recast the tomographic problem in a Bayesian formalism, and obtain qualitative uncertainty estimation on synthetic models, by a low-rank approximation of the posterior covariance matrix, thanks to an ensemble representation.

In this study, after a summary of our ETKF-FWI methodology, we present for the first time a field-data application of this strategy on a 2D line from the Ocean-Bottom Cable recordings of the Valhall field, North Sea.

\section{Formulation of the ETKF-FWI scheme}

Let us recall briefly the ETKF-FWI frame described in Thurin et al. (2017). The overall approach is based on an ensemble representation of the solution $\mathbf{m} \in \mathbb{R}^{n \times N_{e}}$ as a collection of $N_{e}$ subsurface velocity models $m^{(i)} \in \mathbb{R}^{n}$ with $\left\{i=1,2, \ldots, N_{e}\right\}$, where the $n$ parameters of each model are assumed to follow a multivariate Gaussian repartition of covariance $\mathbf{P}$ approximated by the ensemble covariance $\mathbf{P}_{e}$,

$$
\mathbf{P}_{e}=\frac{1}{N_{e}-1}(\mathbf{m}-\bar{m})(\mathbf{m}-\bar{m})^{T},
$$

where $\bar{m}$ is the mean of the ensemble $\mathbf{m}$ and the subscript $e$ denotes the ensemble representation. At the end of the ETKF-FWI process $\bar{m}$ is equivalent to the standard FWI solution while the associated covariance $\mathbf{P}_{e}$ is a low-rank representation of the posterior covariance matrix attached to the FWI problem.

The ETKF-FWI algorithm cycles through two steps: the forecast step and the analysis step, following a frequency continuation approach (Bunks et al., 1995) to mimic the usual ETKF dynamic axis. The forecast step, associated with the forecast operator $\mathscr{F}_{k}$, is considered as a frequency domain FWI minimization at a given frequency or frequency band $k$ for a given initial model $m_{k-1}^{a}{ }^{(i)}$,

$$
\mathscr{F}_{k}\left(m_{k-1}^{a}{ }^{(i)}\right)=\underset{m}{\operatorname{argmin}} \frac{1}{2}\left\|d_{c a l, k}(m)-d_{o b s, k}\right\|^{2}=m_{k}^{f^{(i)}} .
$$

In practice, $m_{k-1}^{a}{ }^{(i)}$ is the output model of the previous ETKF-FWI cycle at frequency $k-1$, while $d_{\text {cal }, k}$ and $d_{o b s, k}$ are the calculated and observed complex waveform data at frequency $k$ and $m_{k}^{f^{(i)}}$ is the forecast (or optimized) model given the aforementioned data. The forecast is applied to the $N_{e}$ models independently such that we obtain the forecast ensemble $\mathbf{m}_{k}^{f}$ containing all the optimized models.

The forecast ensemble is then used in the analysis step to compute the analysis ensemble whose mean 


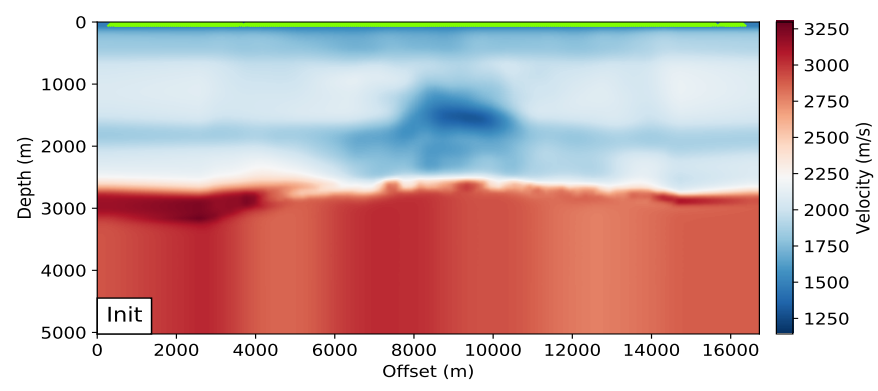

Figure 1 Initial ensemble mean velocity model $m_{0}$. The acquisition location is represented by the light green markers close to the surface.

minimizes the Kalman filter cost function

$$
\mathscr{J}(m)=\frac{1}{2}\left[\left(m-\bar{m}_{k}^{f}\right)^{T}\left(\mathbf{P}_{e, k}^{f}\right)^{-1}\left(m-\bar{m}_{k}^{f}\right)+\left(d_{o b s, k}-\mathscr{H}_{k}\left(\bar{m}_{k}^{f}\right)\right)^{T} \mathbf{R}_{k}^{-1}\left(d_{o b s, k}-\mathscr{H}_{k}\left(\bar{m}_{k}{ }^{f}\right)\right)\right],
$$

where $\mathbf{P}_{e, k}^{f}$ is the covariance matrix attached to the ensemble $\mathbf{m}_{k}^{f}$ through (1), $\mathbf{R}_{k}$ is the measurement noise matrix expressing the data uncertainty, and the observation operator $\mathscr{H}_{k}$ computes and extracts the wavefield at the receiver positions for a frequency $k$ given a model $m^{(i)}$. This cost function contains a data term and a prediction term, similar with the original Kalman Filter idea (Kalman, 1960): finding the best trade-off between the observations (given their errors) and the predictions provided by the forecast (given their errors). The analysis provides the analyzed ensemble $\mathbf{m}_{k}^{a}$, whose distribution is supposed to be a multivariate Gaussian following the analysis covariance matrix $\mathbf{P}_{k}^{a}$, the uncertainty information we are interested in recovering. More details on how the minimization is carried through low-rank representation is detailed in Hunt et al. (2007) for instance. The ETKF-FWI two-fold approach can be summed up as a cycle through two distinct inverse problem: model-wise first, then ensemble-wise.

\section{Application to a 2D OBC line from the Valhall field - set-up}

The considered field data comes from the shallow-marine offshore Valhall field, Norwegian North Sea (provided by AKERBP). The 2D data-set is extracted from the 3D OBC data processed in Operto et al. (2015), and considers a 2D line over what can be interpreted as a hydrocarbon-charged area and has been referred to as the "gas cloud" in (Sirgue et al., 2010). The considered acquisition setup contains 315 hydrophones receivers and 192 sources (same 2D line as processed in Zhou et al., 2018).

For this application, our ETKF-FWI is applied hierarchically with 6 groups of 3 frequencies each, from $3.56 \mathrm{~Hz}$ up to $7.01 \mathrm{~Hz}$. Different ensemble sizes have been considered. We present in this abstract only the result for $N_{e}=600$ members for the sake of compactness. A 2D frequency-domain VTI anisotropic modeling is used. The noise matrix $\mathbf{R}$ is set according to an estimation of the signal-to-noise ratio for each frequency component, reflecting the confidence we have in each piece of data. The initial ensemble mean $m_{0}$ has been built by travel time tomography (provided by AKERBP) and allows to avoid cycle-skipping at lowest frequency (Fig. 1). Each of the $N_{e}$ ensemble members is generated by applying a spatially correlated random perturbation

$$
m_{0}^{(i)}=m_{0}+\mathscr{G} u^{(i)},
$$

where $u^{(i)} \in \mathbb{R}^{n}$ is a zero-mean uniform random vector and $\mathscr{G}$ is a $2 \mathrm{D}$ Gaussian convolution operator. The correlation length of the Gaussian kernels has been chosen to be consistent with the resolution power at the lowest frequency. The amplitudes of the perturbation have been considered large enough to provide diversity, but mitigating the risk of cycle-skipping (assessed with an Eikonal solver).

\section{Results}

The ensemble mean after the 6 ETKF-FWI cycles is shown in Figure 2a. The ETKF-FWI behaves as expected and provides an improved resolution velocity model, with a clear view of what can be interpreted as hydrocarbon-charged layers, especially down to $2.5 \mathrm{~km}$ depth, where we benefit from both diving waves and reflections. A velocity model obtained by usual FWI with similar inversion setup is shown in Figure $2 b$, leading to comparable results for the two approaches.

We emphasize that the ETKF-FWI result is not limited to the ensemble mean. Instead, the entire ensemble is considered as the solution. Even though we can not explicitly compute the covariance matrix, computing lines, columns or the diagonal (variance) of the covariance are cheap and trivial operations using the ensemble representation and the formula (1). 

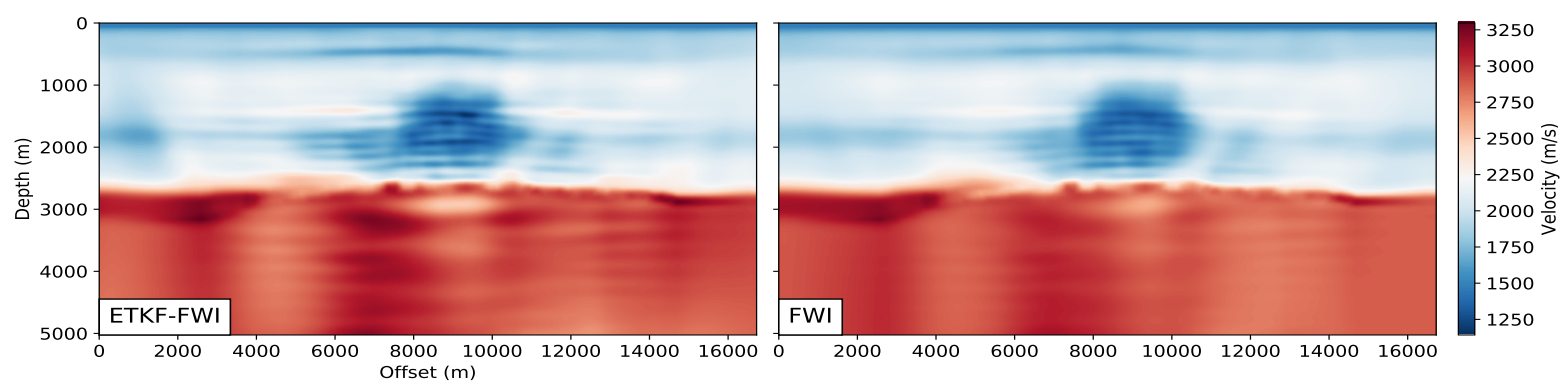

Figure 2 Left : Final ensemble mean after 6 ETKF-FWI cycles from from $3.56 \mathrm{~Hz}$ to $7.01 \mathrm{~Hz}$ with $N_{e}=600$. Right: Comparative FWI result given the same initial model, inversion parameters and observed data.
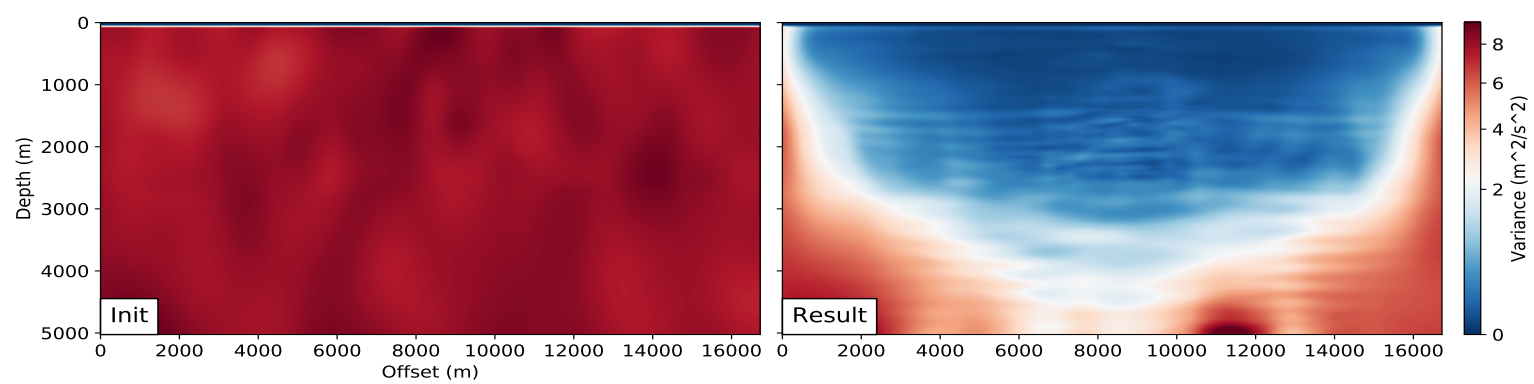

Figure 3 Left : Initial ensemble variance. Note that the water level has not been perturbated. Right : Final ensemble variance after 6 ETKF-FWI cycles from from $3.56 \mathrm{~Hz}$ to $7.01 \mathrm{~Hz}$ with $N_{e}=600$.

We first compute the diagonal of $\mathbf{P}_{e, k}^{a}$, which is the ensemble variance. This variance is mapped as a 2D image. Initial and final variance maps are presented in Figure 3. Variance represents the degree of variability of the solution amongst the ensemble, for each of the parameters: the higher the variance, the greater diversity of solution in the ensemble, which can be related to the solution uncertainty.

After the 6 ETKF-FWI cycles, the final variance values are dominated by the low-wavenumber illumination effect associated with the surface acquisition. The variance is higher on side edges as well as at depth, where only a few waves sample the target, while the well-sampled central shallow region gets weak variance values. Note also how the chalk anticline at $\approx 2.5 \mathrm{~km}$ depth, generating a significant reflector preventing diving waves to penetrate deeper, is also driving the variance.

A high-wavenumber layered structure is also superimposed on the illumination effect of the variance map: higher relative uncertainty structures are encountered at the interfaces of the layered geological formations, coming from the velocity-depth ambiguity and the uncertainties to position velocity-contrast with bandlimited data in uncertain background.

Then, off-diagonal terms of the covariance matrix are extracted as local correlation maps (covariance normalized by its diagonal) and mapped as 2D images for 4 locations (Fig. 4). Correlation maps give a measure of how one parameter is related to its surrounding neighbors, similarly to a point-spread function. The correlation coefficient is a dimensionless measure and goes from -1 (strong negative correlation) to +1 (strong correlation). These correlation maps allow several interesting observations: (1) positive correlations are aligned (mostly horizontally) along the geological structures for almost the four investigated locations. This is expected, as pixels belonging to the same layer should have similar physical parameters. (2) A loss of resolution is observed at depth (magenta parameter for instance) associated with the higher velocity (larger wavelength) and a weaker illumination. (3) Positive correlations are consistent with the recovered geological structures. The magenta parameter is a good example, as the broad positive correlation zone abruptly disappears at the upper boundary of the chalk anticline structure.

\section{Discussion and conclusion}

This study exploits successfully the ETKF-FWI scheme developed and applied to a synthetic case in Thurin et al. (2017). Results on the 2D OBC data from the Valhall field are quite encouraging, with mean, variance and correlation maps being consistent with "usual" FWI and the understanding of the overall FWI process. 

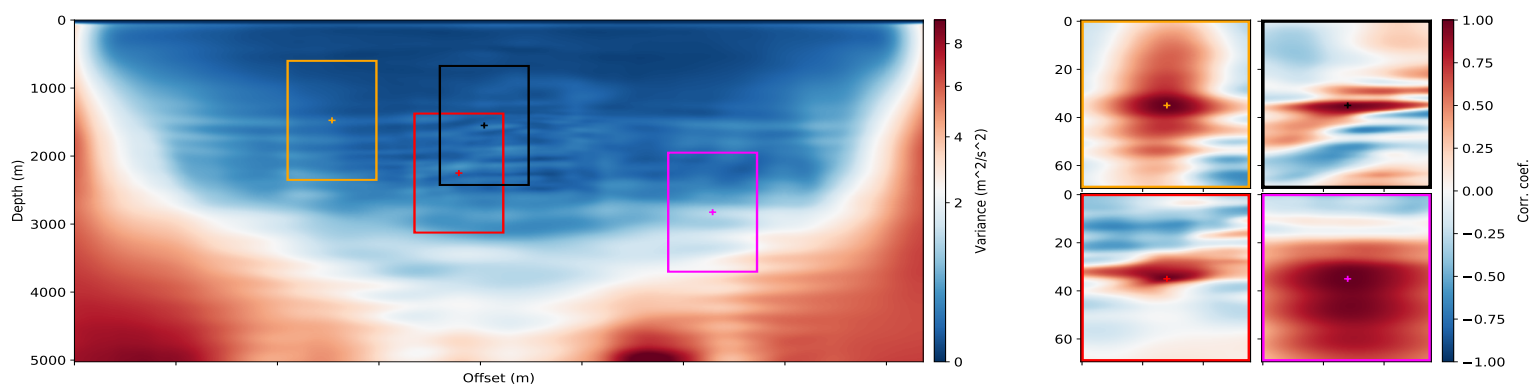

Figure 4 Left: Variance map with four investigated parameters (crosses) and correlation maps subdomains (rectangles). Right: Local correlation maps for the corresponding four parameters.

Although the general setup is close to the previous synthetic case, its challenging nature required a more careful filter setting. The stability of the process is strongly associated with the estimation of the measurement noise matrix $\mathbf{R}$ and the design of the initial ensemble, two key ingredients on field-data applications. Also, despite not being shown here, the ETKF-FWI can suffer from undersampling if the ensemble size is insufficient, leading to bias in the solution. The perspective of this work includes the extension towards multiparameter inversion as-well-as time-domain implementation to assess the feasibility of an ensemble-based approach for larger size FWI applications.

\section{Acknowledgements}

This study was partially funded by the SEISCOPE consortium (http://seiscope2.osug.fr), sponsored by AKERBP, CGG, CHEVRON, EQUINOR, EXXON-MOBIL, JGI, PETROBRAS, SCHLUMBERGER, SHELL, SINOPEC and TOTAL. This study was granted access to the HPC resources of CIMENT infrastructure (https://ciment.ujf-grenoble.fr) and CINES/IDRIS/TGCC under the allocation 046091 made by GENCI. We thank AKERBP ASA and their partner Pandion Energy for providing the dataset and permission to present this work, and the help of Ross Milne from AKERBP.

\section{References}

Bishop, C.H., Etherton, B.J. and Majumdar, S.J. [2001] Adaptive sampling with the ensemble transform Kalman filter. Part I: Theoretical aspects. Monthly weather review, 129(3), 420-436.

Bunks, C., Salek, F.M., Zaleski, S. and Chavent, G. [1995] Multiscale seismic waveform inversion. Geophysics, 60(5), 1457-1473.

Fang, Z., Silva, C.D., Kuske, R. and Herrmann, F.J. [2018] Uncertainty quantification for inverse problems with weak partial-differential-equation constraints. Geophysics, 83(6), R629-R647.

Fichtner, A. and van Leeuwen, T. [2015] Resolution analysis by random probing. Journal of Geophysical Research: Solid Earth, n/a-n/a. 2015JB012106.

Hunt, B., Kostelich, E. and Szunyogh, I. [2007] Efficient data assimilation for spatiotemporal chaos: A local ensemble transform Kalman filter. Physica D: Nonlinear Phenomena, 230(1), 112-126.

Kalman, R. [1960] A new approach to linear filtering and prediction problems. Journal of basic Engineering, 82(1), $35-45$.

Operto, S., Miniussi, A., Brossier, R., Combe, L., Métivier, L., Monteiller, V., Ribodetti, A. and Virieux, J. [2015] Efficient 3-D frequency-domain mono-parameter full-waveform inversion of ocean-bottom cable data: application to Valhall in the visco-acoustic vertical transverse isotropic approximation. Geophysical Journal International, 202(2), $1362-1391$.

Sirgue, L., Barkved, O.I., Dellinger, J., Etgen, J., Albertin, U. and Kommedal, J.H. [2010] Full waveform inversion: the next leap forward in imaging at Valhall. First Break, 28, 65-70.

Tarantola, A. [2005] Inverse Problem Theory and Methods for Model Parameter Estimation. Society for Industrial and Applied Mathematics, Philadelphia.

Thurin, J., Brossier, R. and Métivier, L. [2017] An Ensemble-Transform Kalman Filter - Full Waveform Inversion scheme for Uncertainty estimation. In: 87th SEG Conference and Exhibition 2017, Houston.

Virieux, J., Asnaashari, A., Brossier, R., Métivier, L., Ribodetti, A. and Zhou, W. [2017] An introduction to Full Waveform Inversion. In: Grechka, V. and Wapenaar, K. (Eds.) Encyclopedia of Exploration Geophysics, Society of Exploration Geophysics, R1-1-R1-40.

Virieux, J. and Operto, S. [2009] An overview of full waveform inversion in exploration geophysics. Geophysics, 74(6), WCC1-WCC26.

Zhou, W., Brossier, R., Operto, S., Virieux, J. and Yang, P. [2018] Velocity Model Building by Waveform Inversion of Early Arrivals and Reflections: a 2D Ocean-Bottom-Cable Study with Gas Cloud Effects. Geophysics, 83(2), R141-R157. 